\title{
Estimação de um modelo de ciclos econômicos reais para a emissão de dióxido de carbono no Brasil
}

Cassiano Bragagnolo ${ }^{1}$

Resumo: Este artigo visa propor um modelo teórico simples baseado na teoria RBC que contemple choques de produtividade e choques específicos de emissões, relacionando a emissão de $\mathrm{CO}_{2}$ com outras variáveis econômicas. Uma aplicação para o caso brasileiro, por meio de um método de estimação bayesiano, é apresentada ao final. Os resultados obtidos demonstraram que os choques de produtividade da economia possuem maior influência no modelo do que choques de emissão. Desta forma, um modelo RBC baseado somente nos choques de produtividade da economia poderia representar adequadamente a variância das emissões sem grandes perdas. Observou-se, também, que na presença de choques de produtividade a relação dano-produto tem comportamento pró-cíclico, o que indica que a quantidade de dano ambiental gerado por unidade de produto é maior quando a economia passa por expansões.

Palavras Chave: Emissão de carbono, ciclos econômicos reais, choques na economia

Código JEL: Q20 E32 E37 
BRAGAGNOLO, C. Estimação de um modelo de ciclos econômicos reais para a emissão de dióxido de carbomo no..

Abstract: This article propose a simple theoretical model based on the RBC theory for $\mathrm{CO} 2$ emissions. The model has two different shocks: a productivity shock and a emissions specific shock. At the end, an application for the Brazilian case, through a method of Bayesian estimation, is presented. The results showed that the productivity shocks have more influence in the model than emissions shocks. Thus, a RBC model based only on productivity shocks could satisfactorily represent the variance of emissions without major losses. It was also observed that, in the presence of productivity shocks, environmental damage-output ratio is pro-cyclical, which indicates that the amount of environmental damage generated per unit of output is greater when the economy is in expansion.

\section{Introdução}

Muito embora as teorias econômicas mais populares para estudar problemas ambientais tenham base na teoria microeconômica, a relação entre problemas ambientais e variáveis macroeconômicas agregadas tem sido estudada ao longo do tempo.

A relação entre a evolução do PIB e da emissão de dióxido de carbono é, em geral, bastante proeminente. Doda (2014), por meio de um painel de países membros da OCDE, estudou as propriedades cíclicas da emissão de carbono. Mais especificamente, o autor decompôs as séries em tendência e ciclos utilizando o filtro Hodrick-Prescott (HP). Das séries de ciclos quatro fatos emergiram da análise realizada por Doda (2014):

1. As emissões de dióxido de carbono em um país típico são pró-cíclicas, isto é, apresentam variações em direção compatível com o produto;

2. As emissões de dióxido de carbono dos países analisados são positivamente correlacionadas com o produto;

3. As emissões de dióxido de carbono são mais voláteis que o produto;

4. A volatilidade cíclica das emissões de dióxido de carbono é negativamente correlacionada com o produto per capita.

Inspecionando-se os dados brasileiros pode-se verificar, conforme Figura 1, a grande relação entre os dados anuais de PIB medido em reais de 2013, divulgados pelo IBGE, e a emissão de dióxido de carbono, divulgada pelo Banco Mundial, para o período compreendido entre 1960 e 2010. A correlação entre as duas séries é bastante elevada, da ordem de 98,7\%. 
FIGURA 1 - EVOLUÇÃO DA EMISSÃO DE DIÓXIDO DE CARBONO E DO PIB BRASIL - 1960 A 2010 - EM ÍNDICE (1960=1).

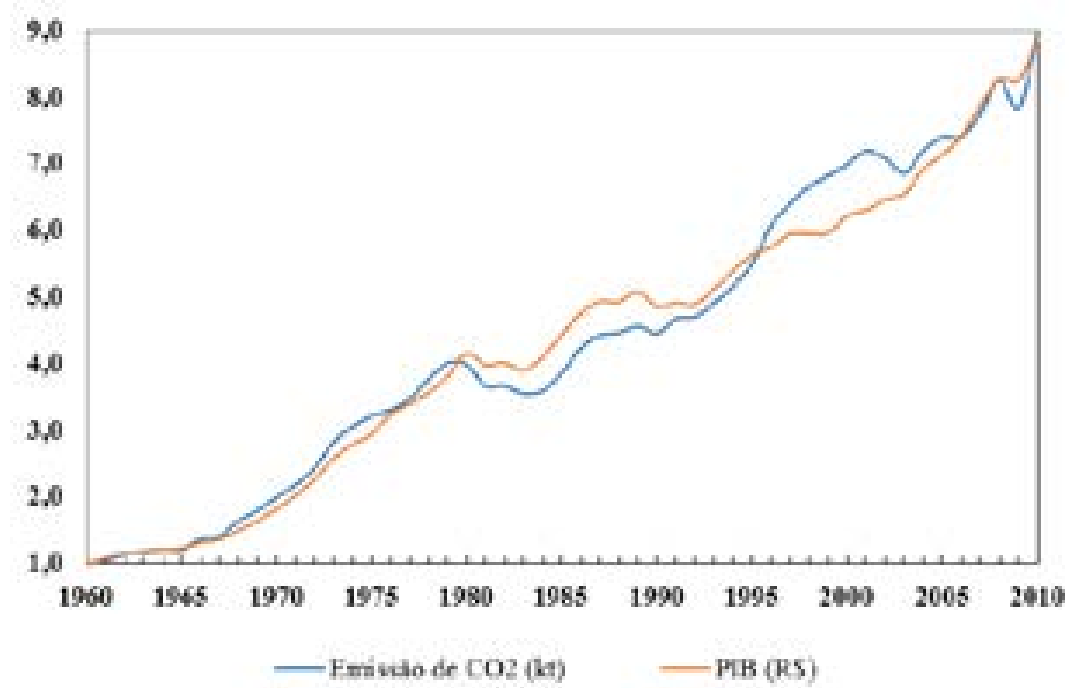

Fonte: Banco Mundial (2015) e Instituto Brasileiro de Geografia e estatística - IBGE (2015)

Na Figura 2 apresenta-se a evolução dos componentes cíclicos das séries de PIB e emissão de dióxido de carbono anuais, filtradas por meio do Filtro HP. ${ }^{1}$ Mesmo quando se avaliam somente os ciclos das séries anuais, eliminando-se as tendências, a correlação é alta, da ordem de $73,4 \% .^{2}$ Este resultado demonstra que os fatos 1 e 2 destacados por Doda (2014) estão presentes nas séries de emissão do Brasil.

O componente cíclico das emissões de dióxido de carbono para o Brasil apresentou desvio padrão em relação à média de 5,0\% para os dados anuais, enquanto que para o produto foi de $3,3 \%$. Assim, o fato 3 destacado por Doda (2014), também pode ser verificado para as séries brasileiras. ${ }^{3}$

1 Os dados foram filtrados utilizando-se o filtro Hodrick-Prescott (HP), procedimento corriqueiro na literatura de ciclos econômicos para separar a tendência dos desvios em torno da tendência. $\mathrm{O}$ valor definido para o parâmetro de suavização da série () foi de 100, valor este comumente utilizado para séries anuais. 2 Heutel (2012) relata um valor de 56,3\% para os EUA com dados trimestrais compreendendo o período de 1981 a 2003.

3 Heutel (2012), com dados trimestrais para os EUA compreendendo o período de 1981 a 2003, relata desvios de $1,3 \%$ para o produto e 2,0\% para a emissão de carbono. 
FIGURA 2 - EVOLUÇÃO DO COMPONENTE CÍCLICO* DA EMISSÃO DE DIÓXIDO DE CARBONO E DO PIB - BRASIL - EM ÍNDICE (1960=1).

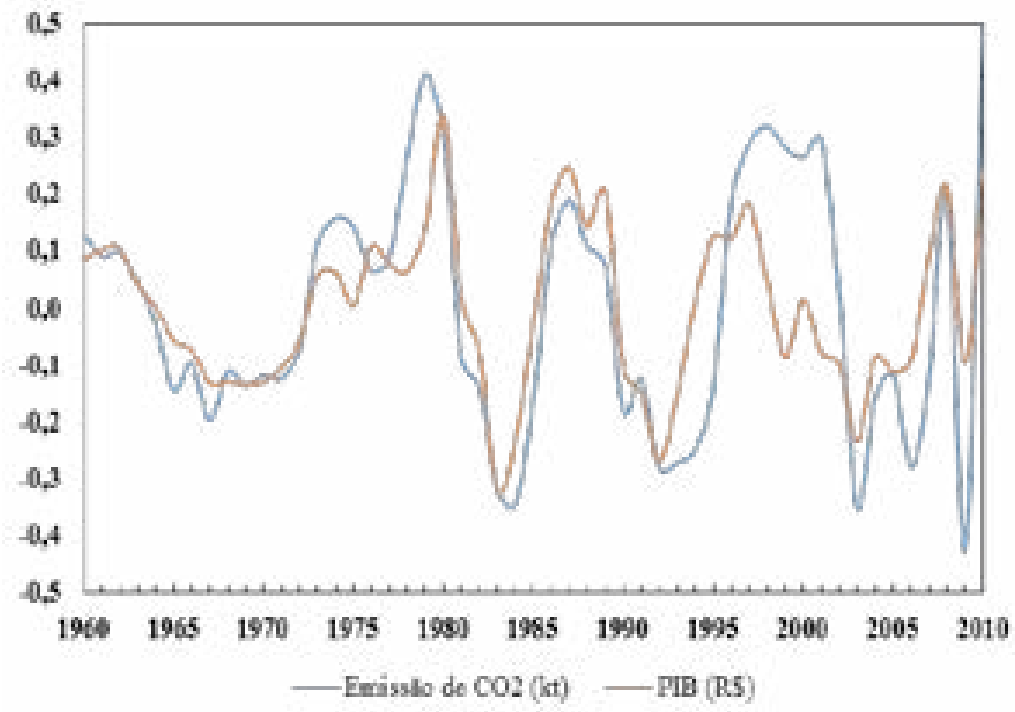

* referem-se a dados filtrados pelo filtro HP com parâmetro de suavização da série $(\lambda)$ de 100.

Fonte: Banco Mundial (2015) e Instituto Brasileiro de Geografia e estatística - IBGE (2015)

Quanto ao fato 4 exposto por Doda (2014), conforme pode ser visto na Tabela 1, não existe uma relação negativa entre a volatilidade das emissões de dióxido de carbono e PIB per capita. A relação para as séries brasileiras é inversa à encontrada para a amostra de países considerados pelo autor. Assim, no Brasil, a medida que o PIB per capita cresceu ao longo do tempo, as emissões se tornaram mais voláteis. 
TABELA 1 - DESVIO PADRÃO CÍCLICO DAS EMISSÕES DE DIÓXIDO DE CARBONO E PIB PER CAPITA.

\begin{tabular}{l|c|c}
\hline Período & $\begin{array}{c}\text { Desvio padrão } \\
\text { cíclico das } \\
\text { emissões de CO2 }\end{array}$ & $\begin{array}{c}\text { PIB per capita } \\
\text { (mil R\$ de 2013) }\end{array}$ \\
\hline $1960-1969$ & 0,118 & 8,080 \\
$1970-1979$ & 0,169 & 13,816 \\
$1980-1989$ & 0,218 & 17,315 \\
$1990-1999$ & 0,254 & 18,058 \\
$2000-2010$ & 0,297 & 20,715 \\
\hline
\end{tabular}

Fonte: Fonte: Banco Mundial (2015) e Instituto de Pesquisa Econômica Aplicada - IPEA (2015)

A relação mais conhecida entre variáveis econômicas agregadas e problemas ambientais é a descrita pela curva conhecida como Curva Ambiental de Kuznets (CAK). O conceito de CAK surgiu no início da década de 1990 e busca entender a relação entre crescimento econômico e o nível de emissão de poluentes de um país. Uma vasta literatura baseada na CAK pode ser encontrada. De acordo com os estudos mais antigos desta literatura, como Holtz-Eakin e Selden (1995) e Schmalensee et al. (1998), espera-se, em geral, que o comportamento da CAK seja de uma curva em formato de "U" invertido. Isto implicaria dizer que nos primeiros estágios de desenvolvimento a degradação ambiental é inevitável, porém ao passo que a renda cresce seriam gerados incentivos para melhorar a qualidade ambiental, fazendo os níveis de degradação ambiental decrescer. Portanto, conforme eleva-se o nível de renda a taxa de crescimento das emissões deve, em um primeiro momento, aumentar. Em um segundo momento, surgem novos fatores como restrições institucionais relativas à questão ambiental que passam a fazer efeito, havendo redução relativa das emissões. Embora estes autores mais antigos que estimaram a CAK encontrem uma curva com forma de "U invertido", vários autores como De Bruyn et al. (1998) e Maddison (2006) encontram outros formatos de curva, enquanto autores como Aldy (2006) e Wagner (2008), contestam a validade da CAK e das técnicas econométricas encontradas na sua obtenção.

Alguns trabalhos têm, recentemente, feito uso de modelos baseados na teoria de ciclos econômicos reais (RBC) visando estudar problemas ambientais. $\mathrm{O}$ tema mais recorrente destes trabalhos consiste na modelagem da emissão de dióxido de carbono. Dentre estes trabalhos pode-se citar Heutel (2012), Fischer e Springborn (2011), Angelopoulos et al. (2010) ${ }^{4}$ e, especificamente

4 Uma discussão mais ampla destes e outros trabalhos sobre o tema pode ser encontrada em Heutel e Fischer (2013) 
para o caso brasileiro, Leal et al. (2015)

O modelo proposto por Heutel (2012) toma como base a teoria RBC para analisar como políticas ambientais respondem aos ciclos econômicos nos Estados Unidos. Para tanto, o autor inclui uma externalidade no seu modelo para representar a emissão de dióxido de carbono do país em questão. Quando comparado aos modelos RBC tradicionais, quatro modificações principais realizadas pelo autor podem ser destacadas. A primeira modificação consiste na eliminação do fator trabalho da função de produção, fazendo com que esta apresente apenas um fator de produção: o capital. A segunda modificação é a inclusão de uma variável para captar o custo de redução das emissões, que depende do percentual de redução e do produto. A terceira modificação é a inclusão de uma restrição para os níveis de emissão do país, que também é dependente do percentual de redução e do produto. Por fim, o modelo inclui uma restrição quanto à acumulação mundial de gases de efeito estufa ao longo do tempo. Heutel (2012) destaca que a luz de seu modelo o nível de emissões de dióxido carbono é pró-cíclico, ainda que na presença de políticas de redução das emissões por meio da cobrança de taxas sobre emissão ou sobre uma cota fixa de poluição.

Fischer e Springborn (2012), por sua vez, propõem a comparação de três diferentes tipos de políticas para redução das emissões, avaliando como estas respondem a choques de produtividade. À Exemplo de Heutel (2012), o modelo utilizado é baseado na teoria RBC, porém a modelagem utilizada difere bastante da proposta por este autor. Fischer e Springborn (2012) incluem em sua função de produção um terceiro insumo, além do capital e do trabalho. Este terceiro é um insumo intermediário que gera poluição no seu uso. Além disso, os autores incluíram o custo do uso deste insumo poluente na restrição orçamentária. O principal resultado obtido por Fischer e Springborn (2012) é que políticas que implementam metas de emissão variáveis geram maior nível de produto, de capital e de trabalho. Além disso, os custos da política são menores quando comparadas às políticas de cotas de emissão fixa e de cobrança de taxas.

Angelopoulos et. al (2010) propuseram outro modelo RBC para as emissões de carbono. Os autores analisam três diferentes tipos de política. A diferença mais acentuada entre o modelo de Angelopoulos et. al (2010) e os demais é a inclusão de dois choques exógeno. Além do choque de produtividade padrão dos modelos RBC, o qual o autor chamou de "economic uncertainty" que poderia ser traduzido como "incerteza econômica", foi incluído um outro choque para captar as variações na relação emissão-produto, o qual os autores denominam de "environmental uncertainty" ou "incerteza ambiental".

Leal et. Al. (2015) propõem um modelo de emissões de $\mathrm{CO}_{2}$ para o caso brasileiro. A análise dos autores se baseia em um modelo similar ao sugerido por Heutel (2012). Os resultados indicaram que o custo de remediação dos impactos das emissões de carbono é mais baixo que o da poluição no Brasil. De 
acordo com os autores este fato justificaria a adoção de políticas que restringem as emissões. Além disso, a exemplo de Heutel (2012), o comportamento ótimo observado por Leal et al. (2015) contemplaria uma política pró-cíclica, na qual o imposto e o teto de emissões diminuiriam em períodos de crise e se elevariam em períodos de expansão.

Os trabalhos enumerados podem ser considerados um ponto de partida para uma literatura de economia ambiental baseada em modelos de ciclos econômicos reais. Por se tratar de uma literatura emergente, inúmeras possíveis extensões podem ser propostas aos modelos disponíveis na literatura.

Assim, este artigo tem como objetivo propor um modelo teórico simples baseado na teoria RBC que relacione a emissão de dióxido de carbono com outras variáveis econômicas de um modelo RBC clássico de trabalho divisível do tipo Hansen (1985). Para ilustrar o modelo apresentado, uma aplicação para o caso brasileiro é proposta utilizando-se de um método de estimação bayesiano do modelo RBC teórico apresentado.

Este artigo é composto de 6 seções. Na segunda seção apresenta-se o modelo teórico $\mathrm{Na}$ terceira apresenta-se, brevemente, o modelo econométrico. $\mathrm{Na}$ quarta seção são apresentadas a base de dados, a estratégia de calibração do modelo e as de prioris. Na quinta seção apresentam-se os resultados e a discussão. Por fim, ao final, na sexta seção apresentam-se as considerações finais.

\section{Modelo Teórico}

Nesta seção apresenta-se o modelo RBC proposto. A diferença entre este modelo e o modelo básico RBC de Hansen (1985) com trabalho divisível é a inclusão de uma curva de Kuznets ambiental para relacionar o nível de produto com a poluição gerada e uma função de dano para transformar a poluição em valores monetários. Além disso, o valor monetário do dano ambiental causado pela poluição foi incluído na restrição orçamentária do problema.

Como no modelo básico de Hansen (1985), com trabalho divisível, procura-se uma sequência de valores que maximize a esperança do valor presente do fluxo de consumo dado pela eq. (1).

$$
\max \sum_{\mathrm{t}=\mathrm{o}}^{\infty} \beta^{\mathrm{t}}\left[\ln \mathrm{c}_{\mathrm{t}}+\mathrm{A} \ln \left(1-\mathrm{h}_{\mathrm{t}}\right)\right]
$$


A variável $\mathrm{c}_{\mathrm{t}}$ é o consumo no tempo $t, \mathrm{~h}_{\mathrm{t}}$ é o tempo trabalhado e $k_{t}$ ó estoque de capital, com o parâmetro $\mathrm{A}>\mathrm{O}$.

Admite-se que o produto é proveniente de uma função de produção do tipo Cobb-Douglas com retornos constantes à escala. A função de produção tem como insumos o capital e o trabalho. As flutuações do produto são provenientes de choques tecnológicos. O produto é dado pela eq. (2).

$$
\mathrm{y}_{\mathrm{t}}=\mathrm{f}\left(\mathrm{z}_{\mathrm{t}}, \mathrm{k}_{\mathrm{t}}, \mathrm{h}_{\mathrm{t}}\right)=\mathrm{e}^{\mathrm{zt}} \mathrm{k}_{\mathrm{t}}{ }^{\theta} \mathrm{h}_{\mathrm{t}}{ }^{1-\theta}
$$

Na qual, a variável $k_{t}$ é o estoque de capital, $h_{t}$ é o trabalho e $z_{t}$ é um choque tecnológico invariante. $\mathrm{O}$ choque tecnológico $(\mathrm{z})$ segue um processo autorregressivo AR(1) definido pela eq. (3).

$$
\mathrm{z}_{\mathrm{t}+1}=\rho \mathrm{z}_{\mathrm{t}}+\mathrm{e}_{\mathrm{t}+1}
$$

Na eq. (3), e $e_{t}$ segue uma distribuição de probabilidades conhecida com média zero.

Assumindo-se que o mercado dos fatores é competitivo, têm-se as condições estabelecidas pelas eq. (4) e (5) para os salários $\left(\mathrm{w}_{\mathrm{t}}\right)$ e renda sobre o capital $\left(r_{t}\right)$.

$$
\begin{aligned}
& \mathrm{r}_{\mathrm{t}}=\theta \mathrm{e}^{\mathrm{zt}} \mathrm{k}_{\mathrm{t}}{ }^{\theta-1} \mathrm{~h}_{\mathrm{t}}{ }^{1-\theta} \\
& \mathrm{w}_{\mathrm{t}}=(1-\theta) \mathrm{e}^{\mathrm{zt}} \mathrm{k}_{\mathrm{t}}{ }^{\theta} \mathrm{h}_{\mathrm{t}}{ }^{-\theta}
\end{aligned}
$$

O capital segue a expressão de acumulação padrão dos modelos RBC dada pela eq. (6) a seguir.

$$
\mathrm{k}_{\mathrm{t}+1}=(1-\delta) \mathrm{k}_{\mathrm{t}}+\mathrm{i}_{\mathrm{t}}
$$

Em que $\mathrm{k}_{\mathrm{t}}$ é o estoque de capital, $\mathrm{i}_{\mathrm{t}}$ é o investimento e $\delta$ é a taxa de depreciação do capital.

A poluição gerada na produção depende do produto e é descrita por uma função cúbica, conforme eq. (7). ${ }^{5}$ 
BRAGAGNOLO, C. Estimação de um modelo de ciclos econômicos reais para a emissão de dióxido de carbomo no...

$$
\mathrm{p}_{\mathrm{t}}=\alpha_{1} \mathrm{y}_{\mathrm{t}}+\alpha_{2}\left(\mathrm{y}_{\mathrm{t}}\right)^{2}+\alpha_{3}\left(\mathrm{y}_{\mathrm{t}}\right)^{3}+\mathrm{j}_{\mathrm{t}}
$$

$\mathrm{j}_{\mathrm{t}}$ é um choque de emissão invariante específico, que segue um processo autorregressivo $\operatorname{AR}(1)$ definido pela eq. (8). ${ }^{6}$

$$
\mathbf{j}_{\mathrm{t}+1}=\gamma \mathbf{j}_{\mathrm{t}}+\mathbf{u}_{\mathrm{t}+1}
$$

O choque $u_{t}$ segue uma distribuição conhecida com média zero.

Para transformar a poluição gerada pela economia $\left(\mathrm{p}_{\mathrm{t}}\right)$ em danos medidos em unidade monetária, utiliza-se a relação linear dada pela eq. (9).

$$
\mathrm{d}_{\mathrm{t}}=\mathrm{Bp}_{\mathrm{t}}
$$

em que $0<B<1$.

Considerando os danos causado pela emissão de poluentes, a economia se depara com a restrição orçamentária descrita pela eq. (10).

$$
\mathrm{k}_{\mathrm{t}+1}-(1-\delta) \mathrm{k}_{\mathrm{t}}+\mathrm{c}_{\mathrm{t}}+\mathrm{d}_{\mathrm{t}}=\mathrm{y}_{\mathrm{t}}
$$

Assim, a equação de Bellman para o problema e suas restrições podem ser descritas pelas equações a seguir.

$$
\mathrm{V}\left(\mathrm{k}_{\mathrm{t}}, \mathrm{z}_{\mathrm{t}}, \mathrm{j}_{\mathrm{t}}\right)=\max _{\mathrm{k}_{\mathrm{t}+1}, \mathrm{~h}_{\mathrm{t}}}\left\{\operatorname{lnc}_{\mathrm{t}}+\mathrm{A} \ln \left(1-\mathrm{h}_{\mathrm{t}}\right)+\beta \mathrm{E}_{\mathrm{t}}\left[\mathrm{V}\left(\mathrm{k}_{\mathrm{t}+1}, \mathrm{z}_{\mathrm{t}+1}, \mathrm{j}_{\mathrm{t}+1}\right)\right]\right\}
$$

sujeito a

$$
\begin{aligned}
& \mathrm{i}_{\mathrm{t}}+\mathrm{c}_{\mathrm{t}}+\mathrm{d}_{\mathrm{t}}=\mathrm{e}^{2 \mathrm{t}} \mathrm{k}_{\mathrm{t}}{ }^{\theta} \mathrm{h}_{\mathrm{t}}^{1-\theta} \\
& \mathrm{k}_{\mathrm{t}+1}=(1-\delta) \mathrm{k}_{\mathrm{t}}+\mathrm{i}_{\mathrm{t}} \\
& \mathrm{z}_{\mathrm{t}+1}=\rho \mathrm{z}_{\mathrm{t}}+\mathrm{e}_{\mathrm{t}+1} \\
& \mathrm{~d}_{\mathrm{t}}=\mathrm{B}\left(\alpha_{1} \mathrm{y}_{\mathrm{t}}+\alpha_{2}\left(\mathrm{y}_{\mathrm{t}}\right)^{2}+\alpha_{3}\left(\mathrm{y}_{\mathrm{t}}\right)^{3}+\mathrm{j}_{\mathrm{t}}\right) \\
& \mathrm{j}_{\mathrm{t}+1}=\gamma \mathrm{j}_{\mathrm{t}}+\mathrm{u}_{\mathrm{t}+1}
\end{aligned}
$$

6 Este segundo choque guarda alguma relação com o proposto por Angelopoulos et. al (2010), o qual o autor chamou de "incerteza ambiental”, que também é relativo às variações na relação emissão e produto. 
BRAGAGNOLO, C. Estimação de um modelo de ciclos econômicos reais para a emissão de dióxido de carbomo no...

Procedendo-sealgumas substituições a equaçãode Bellman doproblema setorna:

$$
\begin{aligned}
& \mathrm{V}\left(\mathrm{k}_{\mathrm{t}}, \mathrm{z}_{\mathrm{t}}, \mathrm{j}_{\mathrm{t}}\right)=\max _{\mathrm{k}_{\mathrm{t}+1} \mathrm{~h}_{\mathrm{t}}}\left\{\ln \left[\mathrm{e}^{\mathrm{tt}} \mathrm{k}_{\mathrm{t}}^{\theta} \mathrm{h}_{\mathrm{t}}^{1-\theta}-\mathrm{k}_{\mathrm{t}+1}+(1-\delta) \mathrm{k}_{\mathrm{t}}-\mathrm{B}\left(\alpha_{1} \mathrm{e}^{\mathrm{tt}} \mathrm{k}_{\mathrm{t}}^{\theta} \mathrm{h}_{\mathrm{t}}^{1-\theta}+\alpha_{2}\left(\mathrm{e}^{\mathrm{tt}} \mathrm{k}_{\mathrm{t}}^{\theta} \mathrm{h}_{\mathrm{t}}^{1-\theta}\right)^{2}+\alpha_{3}\left(\mathrm{e}^{\mathrm{tt}} \mathrm{k}_{\mathrm{t}}^{\theta} \mathrm{h}_{\mathrm{t}}^{1-\theta}\right)^{3}+\mathrm{j}_{\mathrm{t}}\right)\right]+\right. \\
& \left.\mathrm{Aln}\left(1-\mathrm{h}_{\mathrm{t}}\right)+\beta \mathrm{E}_{\mathrm{t}}\left[\mathrm{V}\left(\mathrm{k}_{\mathrm{t}+1}, \mathrm{z}_{\mathrm{t}+1}, \mathrm{j}_{\mathrm{t}+1}\right)\right]\right\}
\end{aligned}
$$

As condições de primeira ordem do problema produzem duas equações, uma para o capital $\left(\mathrm{k}_{\mathrm{t}+1}\right)$ e outra para o trabalho $\left(\mathrm{h}_{\mathrm{t}}\right)$. As condições de primeira ordem são dadas pelas eq. (11) e (12) a seguir:

$$
\begin{gathered}
\frac{\partial \mathrm{V}\left(\mathrm{k}_{\mathrm{t}}, \mathrm{z}_{\mathrm{t}}, \mathrm{j}_{\mathrm{t}}\right)}{\partial \mathrm{k}_{\mathrm{t}+1}}=0=-\frac{1}{\mathrm{c}_{\mathrm{t}}}+\beta \mathrm{E}_{\mathrm{t}}\left[\mathrm{V}\left(\mathrm{k}_{\mathrm{t}+1}, \mathrm{z}_{\mathrm{t}+1}, \mathrm{j}_{\mathrm{t}+1}\right)\right] \\
\frac{\partial \mathrm{V}\left(\mathrm{k}_{\mathrm{t}}, \mathrm{z}_{\mathrm{t}}, \mathrm{j}_{\mathrm{t}}\right)}{\partial \mathrm{k}_{\mathrm{t}+1}}=\mathrm{O}=\frac{1}{\mathrm{c}_{\mathrm{t}}}\left[\mathrm{w}_{\mathrm{t}}-\mathrm{B} \alpha_{\mathrm{1}} \mathrm{w}_{\mathrm{t}}-\mathrm{B} \alpha_{2}(2-2 \theta)\left(\mathrm{e}^{22 \mathrm{t}} \mathrm{k}_{\mathrm{t}}{ }^{2 \theta} \mathrm{h}_{\mathrm{t}}{ }^{1-2 \theta}\right)\right. \\
\left.-\mathrm{B} \alpha_{3}(3-3 \theta)\left(\mathrm{e}^{3 \mathrm{t} t} \mathrm{k}_{\mathrm{t}}{ }^{3 \theta} \mathrm{h}_{\mathrm{t}}{ }^{2-3 \theta}\right)\right]-\mathrm{A} \frac{1}{1-\mathrm{h}_{\mathrm{t}}}
\end{gathered}
$$

Além das condições de primeira ordem, pode-se obter a partir da equação de Bellman a condição de envelope do teorema de Benveniste-Scheikman para o capital, que é dada pela eq. (13) a seguir

$$
\begin{aligned}
& \frac{\partial \mathrm{V}\left(\mathrm{k}_{\mathrm{t}}, \mathrm{z}_{\mathrm{t}}, \mathrm{j}_{\mathrm{t}}\right)}{\partial \mathrm{k}_{\mathrm{t}+1}}=\frac{1}{\mathrm{c}_{\mathrm{t}}}\left[\mathrm{r}_{\mathrm{t}}-\mathrm{B} \alpha_{1} \mathrm{r}_{\mathrm{t}}-\mathrm{B} \alpha_{2}(2 \theta)\left(\mathrm{e}^{2 \mathrm{zt}} \mathrm{k}_{\mathrm{t}}{ }^{2 \theta-1} \mathrm{~h}_{\mathrm{t}}{ }^{2-2 \theta}\right)\right. \\
& \left.-\mathrm{B} \alpha_{3}(3 \theta)\left(\mathrm{e}^{3 z \mathrm{t}} \mathrm{k}_{\mathrm{t}}{ }^{3 \theta-1} \mathrm{~h}_{\mathrm{t}}^{3-3 \theta}\right)+(1-\delta)\right]
\end{aligned}
$$

Combinando e rearranjando a primeira condição de primeira ordem, eq. (11), com a condição de Benveniste-Scheikman, eq. (13), obtém-se a eq. (14) a seguir.

$$
1=\beta \mathrm{E}_{\mathrm{t}}\left\{\frac{\mathrm{c}_{\mathrm{t}}}{\mathrm{c}_{\mathrm{t}+1}}\left[\mathrm{r}_{\mathrm{t}+1}-\mathrm{B} \alpha_{1} \mathrm{r}_{\mathrm{t}+1}-\mathrm{B} \alpha_{2}(2 \theta)\left(\frac{\mathrm{y}_{\mathrm{t}}^{2}}{\mathrm{k}_{\mathrm{t}}}\right)-\mathrm{B} \alpha_{3}(3 \theta)\left(\frac{\mathrm{y}_{\mathrm{t}}^{3}}{\mathrm{k}_{\mathrm{t}}}\right)+(1-\delta)\right]\right\}
$$


Rearranjando a segunda condição de primeira ordem, eq. (12) tem-se a eq. (15) apresentada abaixo.

$$
\left(1-\mathrm{h}_{\mathrm{t}}\right)\left[\mathrm{w}_{\mathrm{t}}-\mathrm{B} \alpha_{1} \mathrm{w}_{\mathrm{t}}-\mathrm{B} \alpha_{2}(2-2 \theta)\left(\frac{\mathrm{y}_{\mathrm{t}}^{2}}{\mathrm{~h}_{\mathrm{t}}}\right)-\mathrm{B} \alpha_{3}(3-3 \theta)\left(\frac{\mathrm{y}_{\mathrm{t}}^{3}}{\mathrm{~h}_{\mathrm{t}}}\right)\right]=\mathrm{Ac} \mathrm{c}_{\mathrm{t}}
$$

As equações (14) e (15) somadas as equações (2) a (10) compõem as onze equações dinâmicas do modelo para as onze variáveis do modelo $k_{t}, z_{t}, j_{t}$ , $i_{t}, c_{t}, h_{t}, y_{t}, r_{t}, w_{t}, d_{t}$ e $p_{t}$.

\section{Método econométrico}

A estimação do modelo proposto será realizada via métodos bayesianos com base nas de prioris e por meio das bases de dados selecionadas. O uso de técnicas bayesianas para estimação de modelos DSGE são amplamente discutidas em trabalhos como Rabanal e Rubio-Ramirez (2005), Schorfheide (2000) e Smets e Wouters (2003).

Para proceder a estimação as equações precisam ser linearizadas previamente. Assim, as condições de primeira ordem apresentadas foram linearizadas ao redor do estado estacionário por meio de uma série de Taylor de grau 1.

Os modelos RBC são geralmente analisados por meio de métodos de calibração. De acordo com Canova (200\&), neste processo, os parâmetros são selecionados com o intuito de que os resultados gerados pelo modelo sejam compatíveis com fatos estilizados observado empiricamente. Tais fatos incluem médias de longo prazo, bem como evidências baseadas em microdados. Ainda de acordo com Canova (2007), considera-se a calibração adequada, quando as simulações baseadas no modelo propostos sejam compatíveis com estes fatos observados da economia.

Canova (2007) destaca que na falta de erros de especificação do modelo e na presença de informação abundante sobre as variáveis do modelo, métodos de calibração deveriam gerar resultados compatíveis com métodos econométricos de máxima verossimilhança. Na prática, este fato raramente ocorre. Segundo Canova (2007), estimações de modelos RBC por máxima verossimilhança podem gerar resultados irreais para os parâmetros, considerando que, muitas vezes, existem informações não contidas na amostra.

Canova (2007) também destaca que os métodos Bayesianos possibilitam que informações de estudos preexistentes na literatura, base de dados adicionais e 
fundamentação em microdados sejam incorporadas nas de priori que servem para reduzir o peso da função de verossimilhança no caso em que os cálculos para os parâmetros não estejam de acordo com as informações adicionais. Neste sentido, de acordo com Lubik e Shorfheide (2007) uma de priori com variação limitada demonstra que o conhecimento sobre o parâmetro a ser estimado é grande. Ainda de acordo com os autores, caso haja evidências inconclusivas sobre um determinado parâmetro, por outro lado, esta incerteza é incorporada na de priori que poderá assumir valores definidos por um intervalo pouco restritivo. A incorporação das incertezas nas de priori permite que se inclua no modelo a incerteza quanto aos parâmetros escolhidos, o que não seria possível fazendo uso de técnicas de calibração.

\section{Base de dados, calibração e estimação do modelo}

A seguir apresentam-se a base de dados utilizada para estimar o modelo, os parâmetros de calibração do estado estacionários e as de prioris bayesianas utilizadas na estimação.

\subsection{Base de dados}

A periodicidade usual dos dados para se estudar modelos de ciclos reais é trimestral. Optou-se por um modelo anual, devido à um problema de disponibilidade de dados. Para o caso do Brasil, séries anuais têm sido empregadas por vários autores, dentre os quais pode-se destacar Ellery, Gomes e Sachsida (2002) e Araújo, Cunha e Carpena (2008).

O período de início e fim das séries ficaram restritos à disponibilidade de dados para as variáveis de interesse da pesquisa. Para as séries utilizadas na estimação o período foi o compreendido entre 1960 e 2010. Os dados foram filtrados utilizando-se o filtro Hodrick-Prescott (HP), procedimento padrão na literatura de ciclos econômicos. $O$ valor para o parâmetro de suavização da série utilizado $(\lambda)$ foi de 100 , valor este comumente utilizado para séries anuais.

Os dados de emissão de dióxido de carbono a serem utilizados na estimação do modelo são os disponibilizados na página eletrônica do Banco Mundial para o Brasil, que se referem as emissões de dióxido de carbono, medidos em milhões 
de toneladas para o período compreendido entre os anos de 1960 e 2010.7

A informação para o PIB utilizados neste trabalho foram os dados anuais deflacionados para 2013 divulgados pelo Instituto Brasileiro de Geografia e Estatística - IBGE para o mesmo período da série de emissão, compreendido entre os anos de 1960 e 2010.

\subsection{Calibração}

Neste trabalho, alguns parâmetros foram selecionados a partir dos resultados de pesquisas existentes na literatura ou calculados a partir de dados básicos, enquanto outros foram calibrados de modo que o estado estacionário determinístico do modelo fosse compatível com fatos da economia brasileira.

O valor usualmente utilizado para a participação do capital na renda $(\theta)$ na economia brasileira é de 0,5 (ver, por exemplo, Bacha e Bonelli, 2004). Ellery, Gomes e Sachsida (2002) utilizaram um valor bastante próximo a este, de o,49, baseado na série de remuneração disponível nas contas nacionais. Kanckzuck e Faria-Jr (2000) calcularam a fração correspondente à remuneração do capital na produção industrial para o período entre 1990 e 1997, encontrando um valor de o,66. Entre outros argumentos, os autores justificam este alto valor encontrado pelo fato da produção industrial ser mais intensiva em capital que a economia como um todo. Neste trabalho optou-se por utilizar um valor para a participação do capital na renda $(\theta)$ de 0,5 .

A depreciação do capital $(\delta)$ utilizada foi de $11 \%$. Este valor foi escolhido visando gerar uma relação estoque de capital e produto próxima à observada empiricamente, que é de cerca de 3 para a economia brasileira.

Para a calibração dos parâmetros de substituição temporal do consumo $(\beta)$ utilizou-se o valor de 0,95. Este valor é compatível com vários modelos para dados anuais disponíveis na literatura para o caso de trabalho divisível.

O valor utilizado para a calibração da constante normalizadora da utilidade (A) foi de 1,7. Este valor é compatível com vários trabalhos presentes na literatura e tem se constituído em um valor padrão para o cálculo de modelos RBC com trabalho divisível.

Para o parâmetro de persistência do choque de produtividade $(\rho)$ utilizou-se o valor o,6. Este valor é muito próximo ao proposto por Ellery, Gomes e Sachsida (2002), 0,589, para a economia brasileira em um modelo anual e 
é compatível com o cálculo do resíduo de Solow para a economia brasileira.

O trabalho de Ellery, Gomes e Sachsida (2002), também serviu como base para a definição da variância do choque de produtividade. O valor estipulado para o parâmetro foi de $0,05 .^{8}$

Para calibrar a equação da emissão de poluentes na economia utilizou-se uma formulação similar a curva ambiental de Kuznets (CAK). O modelo básico estimado foi uma função cúbica do produto ou renda explicando as taxas de emissão de dióxido de carbono, conforme a equação $\mathrm{CO}_{2 \mathrm{t}}=\alpha_{1} \mathrm{y}_{\mathrm{t}}+\alpha_{2} \mathrm{y}_{\mathrm{t}}^{2}+\alpha_{3} \mathrm{y}_{\mathrm{t}}^{3}+\varepsilon_{\mathrm{t}}$, em que y é a renda nacional brasileira no período $t$ e $\mathrm{CO}_{2 t}$ é a emissão de dióxido de carbono no período $t$. Cabe ressaltar que os parâmetros $\alpha_{1}, \alpha_{2}$ e $\alpha_{3}$ da CAK precisaram ser reescalonados, uma vez que o valor para o produto no modelo, bem como a emissão de carbono é expressa em unidades arbitrárias. ${ }^{9}$ Desta forma, os parâmetros foram escalonados para gerar uma relação entre a emissão de carbono e produto comparável com a observada empiricamente.

O carbono gerado pela economia foi transformado em unidades monetárias de acordo com a função de dano proposta na eq. (9). Assim, para o valor inicial do parâmetro seguiu-se o valor proposto pelo Banco Mundial, de que o dano era equivalente a US $\$ 20$ por tonelada de dióxido de carbono emitido no ano de 1995. Este valor foi corrigido para o ano de 2013 e ajustado para R \$ milhões/ mil toneladas, gerando o valor de 0,031 apresentado na Tabela 2.

O valor para a persistência do choque de emissão foi obtido a partir da regressão dos resíduos defasados pelos resíduos obtidos na regressão de estimação da CAK. ${ }^{10}$ Seguindo este procedimento, encontrou-se um valor de 0,89 para o parâmetro $\gamma$.

Os desvios padrões dos erros da regressão dos resíduos da CAK deram origem aos choques de emissão de dióxido de carbono para a economia brasileira. Os valores obtidos para este desvio padrão foram de 0,015.

Na Tabela 2 são apresentados o resumo dos parâmetros utilizados no cálculo do modelo.

8 Ellery Gomes e Sachsida (2002) propõem um valor para o desvio padrão deste choque um pouco inferior de $0,0446$.

9 O procedimento utilizado equivale ao proposto por Heutel (2012) para reescalonar os valores de uma função similar.

10 Neste caso um reescalonamento dos valores dos resíduos foi feito para que sua magnitude fosse compatível com os valores de estado estacionário do modelo. 
BRAGAGNOLO, C. Estimação de um modelo de ciclos econômicos reais para a emissão de dióxido de carbomo no...

TABELA 2 - RESUMO DOS PARÂMETROS OBTIDOS NA LITERATURA

\begin{tabular}{l|c|c}
\hline Parâmetro & Valor & Fonte/critério \\
\hline$\theta$ & 0,5 & $\begin{array}{c}\text { Bacha e Bonelli (2004) } \\
\text { Araújo e Ferreira (1999) } \\
\text { Ellery, Gomes e Sachsida (2002) }\end{array}$ \\
\hline$\delta$ & $11 \%$ & $\overline{\mathrm{k}} / \overline{\mathrm{y}} \cong 3$ \\
\hline$\beta$ & 0,95 & - \\
\hline $\mathrm{A}$ & 1,7 & - \\
\hline$\alpha_{1}$ & 0,393 & $\mathrm{CAK}$ reescalonada $-\overline{\mathrm{d}} / \overline{\mathrm{y}} \cong 1,5 \%$ \\
\hline$\alpha_{2}$ & 0,040 & $\mathrm{CAK}$ reescalonada $-\overline{\mathrm{d}} / \overline{\mathrm{y}} \cong 1,5 \%$ \\
\hline$\alpha_{3}$ & $-0,0124$ & $\mathrm{CAK}$ reescalonada $-\overline{\mathrm{d}} / \overline{\mathrm{y}} \cong 1,5 \%$ \\
\hline$\gamma$ & 0,89 & Resíduo da CAK \\
\hline$\rho$ & 0,6 & Resíduo de Solow \\
\hline $\mathrm{B}$ & 0,031 & Normalização $-\overline{\mathrm{d}} / \overline{\mathrm{y}} \cong 1,3 \%$ \\
\hline$\sigma_{\mathrm{e}}$ & 0,05 & Resíduo de Solow \\
\hline$\sigma_{\mathrm{u}}$ & 0,015 & Resíduo da CAK* \\
\hline
\end{tabular}

* curva ambiental de Kuznets

Fonte: elaboração própria

Uma vez definidos os parâmetros, é possível solucionar o modelo para o estado estacionário. Os valores encontrados para o estado estacionário são apresentados na Tabela 3. 
TABELA 3 - VALORES DO ESTADO ESTACIONÁRIO

\begin{tabular}{l|l}
\hline Variável & Valor \\
\hline Produto (y) & 0,933 \\
Consumo (c) & 0,609 \\
Estoque de capital (k) & 2,832 \\
Investimento (i) & 0,312 \\
Horas de trabalho (h) & 0,308 \\
Emissão de CO2 (p) & 0,392 \\
Dano ambiental gerado pelas emissões de CO2 (d) & 0,012 \\
\hline
\end{tabular}

Fonte: resultados da pesquisa

\subsection{Definição das de prioris}

Nesta seção discutem-se as de prioris utilizadas no cálculo do modelo. Para definir as de prioris é necessário determinar a média, o desvio padrão, a distribuição e o domínio dos parâmetros a serem estimados.

As médias utilizadas são iguais aos parâmetros utilizados para calibrar o estado estacionário. As funções de densidade mais comuns para modelos RBC estimados via métodos bayesianos foram definidas de acordo com trabalhos disponíveis na literatura como Smets e Wouters (2003) e An e Schorfheide (2007).

Optou-se por incluir uma variância mais elevada para as variáveis relacionadas à emissão de dióxido de carbono e ao dano ambiental, uma vez que a definição de seus valores é pouco discutida na literatura. Este procedimento incorpora maior incerteza para estas variáveis quanto aos seus reais valores.

Na Tabela 4 é apresentado o resumo das informações acerca das de prioris para o modelo proposto. 
BRAGAGNOLO, C. Estimação de um modelo de ciclos econômicos reais para a emissão de dióxido de carbomo no...

TABELA 4 - DISTRIBUIÇÕES À PRIORI DOS PARÂMETROS DO MODELO

\begin{tabular}{l|c|c|c|c}
\hline Parâmetro & Domínio & Média & Variância & Densidade \\
\hline$\theta$ & Entre o e 1 & 0,5 & 0,05 & Beta \\
$\delta$ & $\begin{array}{c}\text { Entre 0,01 } \\
\text { e 0,2 }\end{array}$ & 0,11 & 0,05 & Beta \\
$\beta$ & Entre 0.5 e 1 & 0,95 & 0,01 & Beta \\
$\mathrm{A}$ & $\mathrm{R}^{*}+$ & 1,7 & 0,2 & Normal \\
$\alpha_{1}$ & $\mathrm{R}^{*}$ & 0,393 & 1,6 & Uniforme \\
$\alpha_{2}$ & $\mathrm{R}^{*}$ & 0,040 & 0,24 & Uniforme \\
$\alpha_{3}$ & $\mathrm{R}^{*}$ & $-0,0124$ & 0,1 & Uniforme \\
$\gamma$ & Entre o e 1 & 0,89 & 0,3 & Normal \\
$\rho$ & Entre o e 1 & 0,6 & 0,2 & Normal \\
$\mathrm{B}$ & $\mathrm{R}^{*}+$ & 0,031 & 0,2 & Uniforme \\
$\sigma_{\mathrm{e}}$ & $\mathrm{R}^{*}+$ & 0,05 & - & Gama \\
$\sigma_{\mathrm{u}}$ & $\mathrm{R}^{*}+$ & 0,015 & inversa \\
\hline
\end{tabular}

Fonte: elaboração própria

\section{Resultados e discussão}

A estimação do modelo foi feita a partir dos dados apresentados na seção 4.1 para produto $(y)$ e emissões de $\mathrm{CO}_{2}(p)$. O modelo foi estimado, apenas, com base nestas duas variáveis, pois para evitar singularidade estocástica, são necessários número de choques iguais ao número de variáveis observadas (CANOVA, 2007). Conforme já discutido anteriormente, a amostra compreende o período entre 1960 e 2010.

Na Tabela 5 são apresentadas as médias das distribuições de priori e de posteriori dos parâmetros do modelo, bem como o intervalo de confiança de 90\% de posteriori. Os parâmetros que apresentaram maior diferença entre a priori proposta e a de posteriori estimada foram a depreciação do capital $(\beta)$ , o parâmetro de persistência do choque de emissão $(\gamma)$ e o parâmetro que relaciona o dano monetário causado à quantidade de poluição gerada $(\mathrm{B})$. 
TABELA 5 - DE PRIORIS E DE POSTERIORIS

\begin{tabular}{l|c|c|c|c}
\hline Parâmetro & $\begin{array}{c}\text { Média } \\
\text { de priori }\end{array}$ & $\begin{array}{c}\text { Média } \\
\text { de posteriori }\end{array}$ & \multicolumn{2}{|c}{$\begin{array}{c}\text { Intervalo de } \\
\text { confiança } 90 \%\end{array}$} \\
\hline$\theta$ & 0,500 & 0,494 & 0,439 & 0,556 \\
$\beta$ & 0,950 & 0,949 & 0,933 & 0,966 \\
$\delta$ & 0,110 & 0,094 & 0,038 & 0,146 \\
A & 1,700 & 1,657 & 1,326 & 1,986 \\
$\rho$ & 0,600 & 0,582 & 0,420 & 0,758 \\
$\gamma$ & 0,890 & 0,762 & 0,504 & 0,990 \\
$\alpha_{1}$ & 0,393 & 0,408 & 0,271 & 0,546 \\
$\alpha_{2}$ & 0,040 & 0,023 & $-0,243$ & 0,338 \\
$\alpha_{3}$ & $-0,012$ & $-0,012$ & $-0,150$ & 0,159 \\
B & 0,031 & 0,191 & 0,030 & 0,368 \\
\hline
\end{tabular}

Fonte: resultados da pesquisa

Na tabela 6 apresentam-se as médias das de prioris e as médias, os intervalos de confiança das de posterioris para os choques $\left(\sigma_{\mathrm{e}} \mathrm{e} \sigma_{\mathrm{u}}\right)$, donde pode-se observar que os valores propostos de priori possuem valor mais elevado que os choques de posteriori e que esta alteração é mais acentuada para o choque de emissão. 
BRAGAGNOLO, C. Estimação de um modelo de ciclos econômicos reais para a emissão de dióxido de carbomo no...

TABELA 6 - DE PRIORIS E DE POSTERIORIS PARA OS CHOQUES DO MODELO

\begin{tabular}{l|c|c|c|c}
\hline Parâmetro & $\begin{array}{c}\text { Média } \\
\text { de priori }\end{array}$ & $\begin{array}{c}\text { Média de } \\
\text { posteriori }\end{array}$ & \multicolumn{2}{|c}{$\begin{array}{c}\text { Intervalo de confiança } \\
90 \%\end{array}$} \\
\hline $\begin{array}{l}\text { Desvio padrão } \\
\text { do choque de } \\
\text { produtividade }\left(\sigma_{\mathrm{e}}\right)\end{array}$ & 0,050 & 0,0224 & 0,0180 & 0,0265 \\
$\begin{array}{l}\text { Desvio padrão do } \\
\text { choque de emissão }\left(\sigma_{\mathrm{u}}\right)\end{array}$ & 0,015 & 0,0019 & 0,0018 & 0,0021 \\
\hline
\end{tabular}

Fonte: resultados da pesquisa

Na Tabela 7 apresenta-se a decomposição da variância das variáveis do modelo com relação aos choques exógenos de produtividade (e) e de emissão (u). Os resultados mostram que os choques de produtividade apresentam influência mais acentuada sobre as variações de curto prazo na emissão de poluentes do que os choques específicos de emissão de $\mathrm{CO}_{2}$. Assim, pode-se afirmar que um modelo que levasse em consideração somente os choques de produtividade da economia poderia representar de maneira adequada a emissão de $\mathrm{CO}_{2}$ e que a influência de tecnologias específicas de redução de emissões não tem sido relevante para explicar o comportamento de curto prazo dos níveis de emissão.

TABELA 7 - DECOMPOSIÇÃO DA VARIÂNCIA DAS VARIÁVEIS DO MODELO EM RELAÇÃO AOS CHOQUES DE PRODUTIVIDADE (E) E DE EMISSÃ O (U)

\begin{tabular}{l|c|c}
\hline Variável & $\begin{array}{c}\text { Choque de } \\
\text { produtividade (e) }\end{array}$ & $\begin{array}{c}\text { Choque de emissão } \\
\text { (u) }\end{array}$ \\
\hline Produto (y) & $100,00 \%$ & $0,00 \%$ \\
Consumo (c) & $99,96 \%$ & $0,04 \%$ \\
Estoque de capital (k) & $99,99 \%$ & $0,01 \%$ \\
Investimento (i) & $99,99 \%$ & $0,01 \%$ \\
Horas de trabalho (h) & $99,98 \%$ & $0,02 \%$ \\
$\begin{array}{l}\text { Emissão de CO2 (p) } \\
\begin{array}{l}\text { Dano ambiental gerado pelas } \\
\text { emissões de CO2 (d) }\end{array}\end{array}$ & $97,03 \%$ & $2,97 \%$ \\
\hline
\end{tabular}

Fonte: resultados da pesquisa 
As funções de impulso resposta aos choques de produtividade do modelo são apresentados na Figura 3. O dano ambiental possui variação ao redor do seu valor de estado estacionário ligeiramente superior à variação do produto em relação ao estado estacionário. A dissipação do efeito do choque também segue padrão similar.

A variação na relação dano-produto, também, é apresentada na Figura 3 abaixo, na qual é possível perceber uma variação inicial de $0,5 \%$ na relação dano-produto devido ao choque de produtividade na economia. Isto significa dizer que o impacto ambiental das emissões tem padrão pró-cíclico na ausência de qualquer controle ou política ambiental.

As demais variáveis como capital, trabalho e consumo, apresentam comportamento em relação ao choque de produtividade similar ao modelo básico de Hansen (1985) com trabalho divisível.

FIGURA 3 - RESPOSTA A UM CHOQUE DE PRODUTIVIDADE, COM DESVIO PADRÃO UM, MEDIDO EM DESVIO PERCENTUAL EM RELAÇÃO AO ESTADO ESTACIONÁRIO

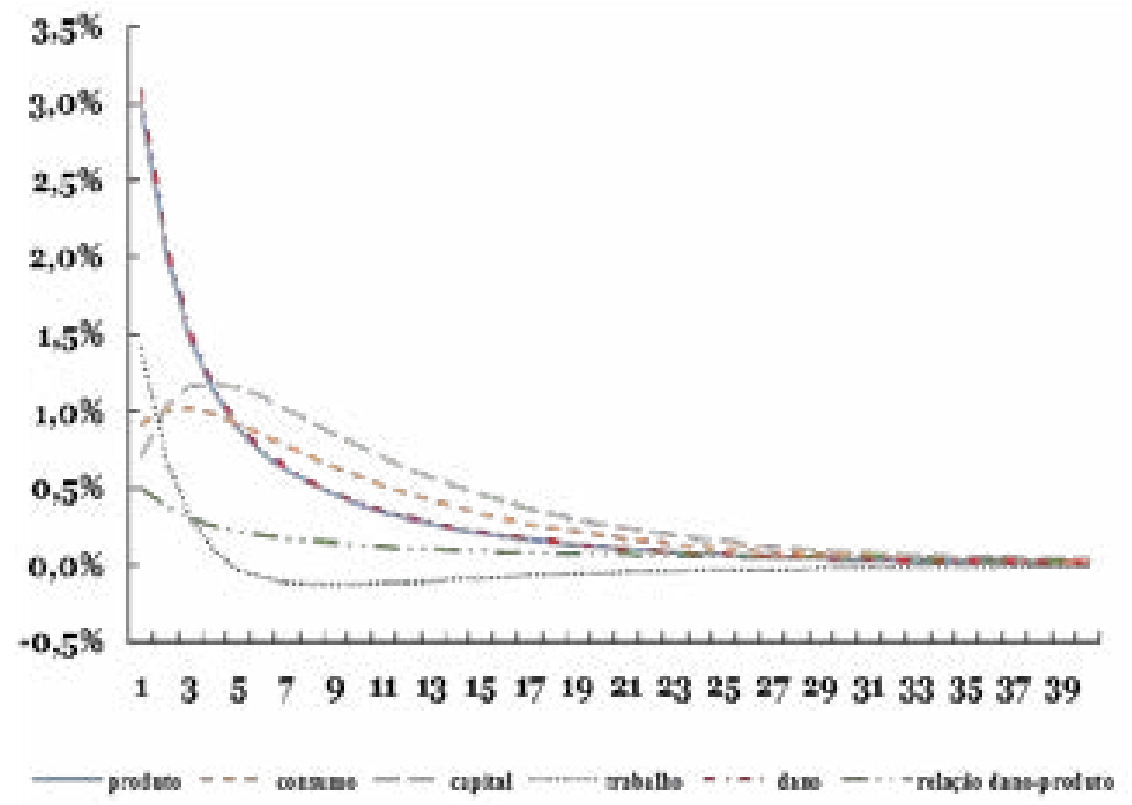

Fonte: resultados da pesquisa

As funções de impulso resposta aos choques de emissão do modelo são 
apresentados na Figuras 4. Neste caso, o dano ambiental possui impacto no seu valor de estado estacionário bastante superior à variação do produto em relação ao estado estacionário.

A variação na relação dano-produto, que também é apresentada na Figura 4, é bastante acentuada, quando comparada a das demais variáveis, inicialmente da ordem de cerca de $0,5 \%$. A dissipação do choque de emissão na relação dano-produto é bem mais lenta daquela observada para o produto.

As demais variáveis como capital, produto, trabalho e consumo, apresentam resposta em relação ao choque de emissão pouco expressivo.

FIGURA 4 - RESPOSTA A UM CHOQUE DE EMISSÃO, COM DESVIO PADRÃO UM, MEDIDO EM DESVIO PERCENTUAL EM RELAÇÃO AO ESTADO ESTACIONÁRIO

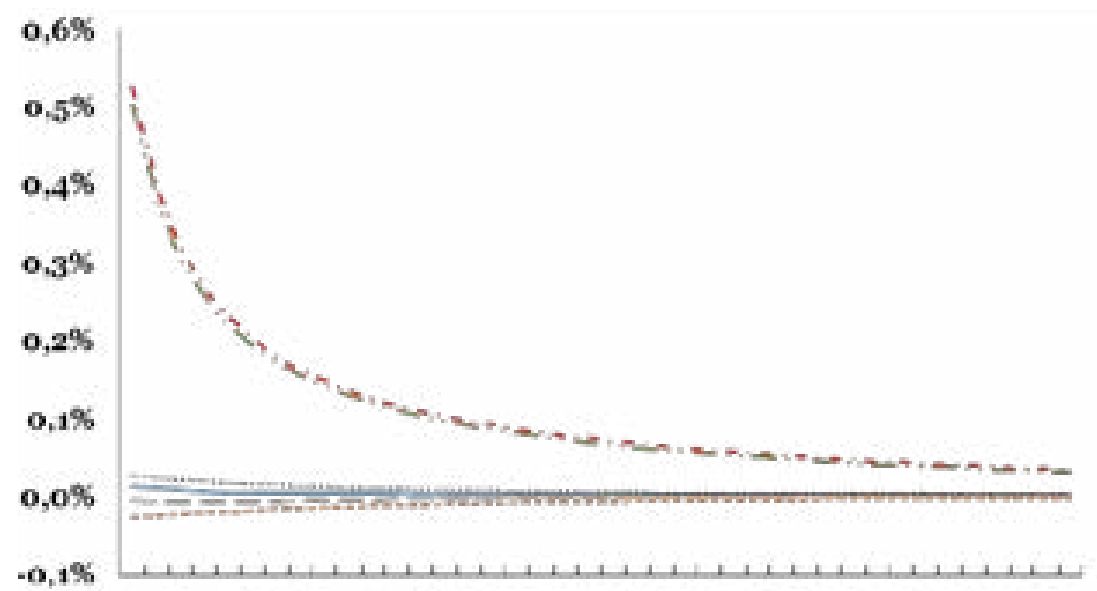

$\begin{array}{llllllllllllllllllll}1 & 3 & 5 & 7 & 9 & 11 & 13 & 15 & 17 & 19 & 21 & 23 & 25 & 27 & 29 & 31 & 33 & 35 & 37 & 39\end{array}$

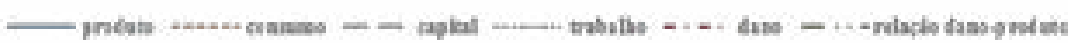

Fonte: resultados da pesquisa

\section{Considerações Finais}

Diferentemente dos demais trabalhos disponíveis na literatura, neste estimou-se parâmetros por meio de métodos bayesianos a partir de um modelo RBC básico de Hansen (1985) com trabalho divisível estendido para analisar a emissão de dióxido de carbono no Brasil. Cabe ressaltar que os parâmetros estimados neste trabalho podem ajudar na calibração e eventual avaliação 
de políticas de futuros trabalhos.

Os resultados obtidos demonstram que os choques de produtividade da economia têm grande influência sobre as variações de curto prazo na emissão de poluentes quando comparados a choques específicos de emissão. Esta afirmação se baseia, primeiramente, nos resultados obtidos para a decomposição da variância das variáveis do modelo que demonstraram que mais de 97\% das variações nas emissões são devidas a choques de produtividade na economia e não choques de emissão. Segundo, o desvio padrão da de priori do choque era muito mais elevado que seu resultado final, o que demonstra impactos menos expressivos que o indicado inicialmente por meio da de priori. Assim, um modelo RBC baseado somente nos choques de produtividade da economia poderia representar adequadamente a variância das emissões sem grandes perdas.

Observou-se, ainda, que na presença de choques de produtividade a relação dano-produto apresentou comportamento pró-cíclico, o que indica que, à luz do modelo, a quantidade de dano ambiental gerado por unidade de produto aumenta à medida que a economia passa por expansões. $\mathrm{O}$ contrário aconteceria no caso de recessões.

Por fim, cabe salientar que a literatura sobre o comportamento das emissões nos ciclos econômicos é recente e crescente e que inúmeras extensões ao modelo proposto, e a outros disponíveis na literatura, podem ser sugeridas. Concomitantemente, outros problemas ambientais poderiam ser estudados fazendo uso de ferramental similar.

\section{Referências Bibliográficas}

Aldy, J. E. (2006). Per capita carbon dioxide emissions: convergence or divergence? Environmental and Resource Economics, 33(4), 533-555.

An, S. \& Schorfheide, F. (2007). Bayesian analysis of DSGE models. Econometric reviews, 26(2-4), 113-172.

Angelopoulos, K. \& Economides, G. \& Philippopoulos, A. (2010). What is the best environmental policy? Taxes, permits and rules under economic and environmental uncertainty.

Araújo, E. \& Carpena, L., \& Cunha, A. B. (2008). Brazilian business cycles and growth from 1850 to 2000. Estudos Econômicos (São Paulo), 38(3), 557-581.

Bacha, E. L. \& Bonelli, R. (2004). Accounting for Brazil's growth experience-1940-2002.

Canova, F. (2007). Methods for applied macroeconomic research (Vol. 13). Princeton University Press.

De Bruyn, S. M. \& Van Den Bergh, J. C., \& Opschoor, J. B. (1998). Economic growth 
BRAGAGNOLO, C. Estimação de um modelo de ciclos econômicos reais para a emissão de dióxido de carbomo no...

and emissions: reconsidering the empirical basis of environmental Kuznets curves. Ecological Economics, 25(2), 161-175.

Doda, B. (2014). Evidence on business cycles and CO2 emissions. Journal of Macroeconomics, 4O, 214-227.

Ellery Jr, R. \& Gomes, V. \& Sachsida, A. (2002). Business cycle fluctuations in Brazil. Revista Brasileira de Economia, 56(2), 269-308.

Fischer, C. \& Springborn, M. (2011). Emissions targets and the real business cycle: Intensity targets versus caps or taxes. Journal of Environmental Economics and Management, 62(3), 352-366.

Hansen, G. D. (1985). Indivisible labor and the business cycle. Journal of monetary Economics, 16(3), 309-327.

Heutel, G. (2012). How should environmental policy respond to business cycles? Optimal policy under persistent productivity shocks. Review of Economic Dynamics, 15(2), 244-264.

Heutel, G. \& Fischer, C. (2013). Environmental macroeconomics: Environmental policy, business cycles, and directed technical change (No. w18794). National Bureau of Economic Research.

Holtz-Eakin, D. \& Selden, T. M. (1995). Stoking the fires? CO2 emissions and economic growth. Journal of public economics, 57(1), 85-101.

Kanczuk, F. \& Faria, F. C. P. (2000). Ciclos reais para a indústria brasileira. Estudos Econômicos, 30(3), 335-350.

Leal, R. A. \& Ely, R. A. \& Uhr, J. G. Z. \& Uhr, D. A. P. (2015). Ciclos econômicos e emissão de $\mathrm{CO} 2$ no Brasil: uma análise dinâmica para políticas ambientais ótimas. Revista Brasileira de Economia, 69(1), 53-73.

Lubik, T. \& Schorfheide, F. (2006). A Bayesian look at the new open economy macroeconomics. In NBER Macroeconomics Annual 2005, Volume 20 (pp. 313-382). MIT Press.

Maddison, D. (2006). Environmental Kuznets curves: A spatial econometric approach. Journal of Environmental Economics and management, 51(2), 218-230.

Rabanal, P. \& Rubio-Ramírez, J. F. (2005). Comparing New Keynesian models of the business cycle: A Bayesian approach. Journal of Monetary Economics, 52(6), 1151-1166.

Schmalensee, R. \& Stoker, T. M. \& Judson, R. A. (1998). World carbon dioxide emissions: 1950-2050. Review of Economics and Statistics, 8O(1), 15-27.

Schorfheide, F. (2000). Loss function-based evaluation of DSGE models. Journal of Applied Econometrics, 15(6), 645-670.

Smets, F. \& Wouters, R. (2003). An estimated dynamic stochastic general equilibrium model of the euro area. Journal of the European economic association, 1(5), 1123-1175.

Wagner, M. (2008). The carbon Kuznets curve: a cloudy picture emitted by bad econometrics? Resource and Energy Economics, 30(3), 388-408. 
